\title{
Transverse Beam Emittance Measurement by Undulator Radiation Power Noise
}

\author{
Ihar Lobach $\odot^{*}$ \\ The University of Chicago, Department of Physics, Chicago, Illinois 60637, USA \\ Sergei Nagaitsev®, ${ }^{\dagger}$ Valeri Lebedev®, Aleksandr Romanov®, Giulio Stancari®, and Alexander Valishev \\ Fermi National Accelerator Laboratory, Batavia, Illinois 60510, USA \\ Aliaksei Halavanau and Zhirong Huang \\ SLAC National Accelerator Laboratory, Stanford University, Menlo Park, California 94025, USA \\ Kwang-Je Kim ${ }^{\dagger}$ \\ Argonne National Accelerator Laboratory, Lemont, Illinois 60439, USA
}

(Received 8 December 2020; accepted 22 February 2021; published 1 April 2021)

\begin{abstract}
Generally, turn-to-turn power fluctuations of incoherent spontaneous synchrotron radiation in a storage ring depend on the $6 \mathrm{D}$ phase-space distribution of the electron bunch. In some cases, if only one parameter of the distribution is unknown, this parameter can be determined from the measured magnitude of these power fluctuations. In this Letter, we report an absolute measurement (no free parameters or calibration) of a small vertical emittance $(5-15 \mathrm{~nm} \mathrm{rms})$ of a flat beam by this method, under conditions, when it is unresolvable by a conventional synchrotron light beam size monitor.
\end{abstract}

DOI: 10.1103/PhysRevLett.126.134802

Most often, noise is encountered in a negative context and is considered something that needs to be minimized. However, there are multiple examples where noise is used as a noninvasive probe into the parameters of a certain system, and even to measure fundamental constants. Examples include the determination of the Boltzmann constant $k_{B}$ by the thermal noise in an electrical conductor [1] and the measurement of the elementary charge $e$ by the shot noise of the electric current in a vacuum tube [2]. In fact, the latter effect is also relevant to accelerators and storage rings, where it is known as Schottky noise [3] due to the finite number of charge carriers in the beam, as described by Schottky [4]. Many beam parameters, such as the momentum spread, the number of particles, and even transverse rms emittances, are imprinted into the power spectrum of Schottky noise. It is often used in beam diagnostics [5-7].

Synchrotron radiation is generated by individual electrons in the beam. Hence, Schottky noise in the beam current must pass on to the synchrotron radiation power in some way. Therefore, one could assume that the synchrotron radiation power noise may carry information about beam parameters as well. This assumption is, in fact,

Published by the American Physical Society under the terms of the Creative Commons Attribution 4.0 International license. Further distribution of this work must maintain attribution to the author(s) and the published article's title, journal citation, and DOI. correct. Three decades ago, Ref. [8] reported the results of an experimental study into statistical properties of wiggler radiation in a storage ring. It was noted that the magnitude of turn-to-turn intensity fluctuations depends on the dimensions of the electron bunch. The potential in beam instrumentation was soon realized [9] and a number of papers followed. However, to this day, mostly measurements of a bunch length via these fluctuations have been discussed [10-12]. Only Ref. [13] reported an order-ofmagnitude measurement of a transverse emittance. In this Letter, we describe a new fluctuations-based technique for an absolute measurement of a transverse emittance. There are no free parameters in our equations, nor is a calibration required. However, the transverse and longitudinal focusing functions of the storage ring are assumed to be known. This technique is tested at the Integrable Optics Test Accelerator (IOTA) storage ring at Fermilab [14]. For a beam with approximately equal and relatively large transverse rms emittances, the results agree with conventional visible synchrotron light monitors (SLMs) [15]. Then, in a different regime, we measure a much smaller vertical emittance of a flat beam, unresolvable by our SLMs. These emittance measurements agree with estimates, based on the beam lifetime. We also discuss possible further improvements.

Let us assume that we have a detector that can measure the number of detected synchrotron radiation photons $\mathcal{N}$ at each revolution in a storage ring. Then, according to Refs. $[8,16-18]$, the variance of this number is 


$$
\operatorname{var}(\mathcal{N})=\left\langle(\mathcal{N}-\langle\mathcal{N}\rangle)^{2}\right\rangle=\langle\mathcal{N}\rangle+\frac{1}{M}\langle\mathcal{N}\rangle^{2},
$$

where the linear term represents the photon shot noise, related to the quantum discrete nature of light. This effect would exist even if there was only one electron, circulating in the ring. Indeed, the electron would radiate photons with a Poisson distribution [19-21]. The quadratic term in Eq. (1) corresponds to the interference of fields, radiated by different electrons. Changes in relative electron positions and velocities, inside the bunch, result in fluctuations of the radiation power and, consequently, of the number of detected photons. In a storage ring, the effect arises from betatron and synchrotron motion, from radiation induced diffusion, etc. The dependence of $\operatorname{var}(\mathcal{N})$ on the $6 \mathrm{D}$ phasespace distribution of the electron bunch is introduced through the parameter $M$, which is conventionally called the number of coherent modes [8,16,17]. In addition to bunch parameters, $M$ depends on the specific spectralangular distribution of the radiation, on the angular aperture, and on the detection efficiency (as a function of wavelength). Previously, we derived an equation for $M$ [[22] Eq. (2)] for a Gaussian transverse beam profile and an arbitrary longitudinal bunch density distribution $\rho(z)$ (normalized), assuming an rms bunch length much longer than the radiation wavelength. In this Letter, $M$ is calculated by this equation numerically, using our computer code [23], as a function of transverse emittances $\epsilon_{x}$ and $\epsilon_{y}$, the rms momentum spread $\sigma_{p}$, and the effective bunch length, $\sigma_{z}^{\text {eff }}=1 /\left[2 \sqrt{\pi} \int \rho^{2}(z) \mathrm{d} z\right]$, equal to the rms bunch length $\sigma_{z}$ for a Gaussian distribution.

For illustration purposes, let us assume a Gaussian spectral-angular distribution for the number of detected photons $\mathcal{N}$, namely,

$$
\frac{\mathrm{d}^{3} \mathcal{N}}{\mathrm{d} k \mathrm{~d} \theta_{x} \mathrm{~d} \theta_{y}}=C \exp \left[-\frac{\left(k-k_{0}\right)^{2}}{2 \sigma_{k}^{2}}-\frac{\theta_{x}^{2}}{2 \sigma_{\theta_{x}}^{2}}-\frac{\theta_{y}^{2}}{2 \sigma_{\theta_{y}}^{2}}\right],
$$

where $k$ is the magnitude of the wave vector, $\theta_{x}$ and $\theta_{y}$ represent the horizontal and vertical angles of the direction of the radiation in the paraxial approximation, $k_{0}$ refers to the center of the radiation spectrum, $\sigma_{k}$ is the spectral rms width, $\sigma_{\theta_{x}}$ and $\sigma_{\theta_{y}}$ are the angular rms radiation sizes, and $C$ is a constant. Then $[10,22]$
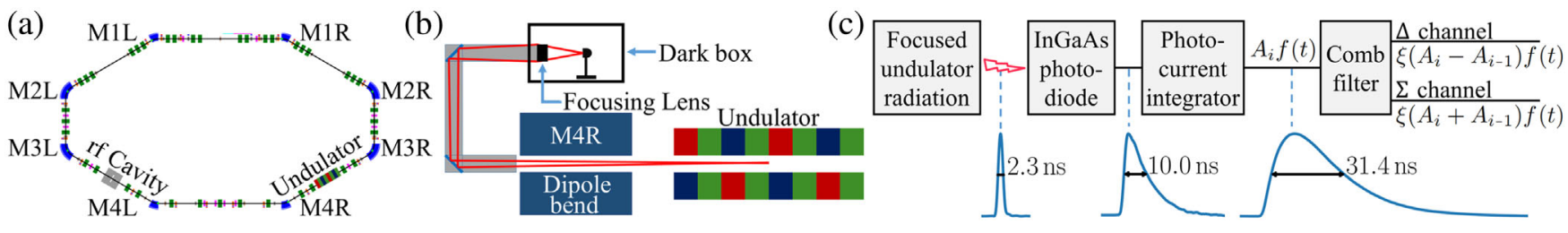

FIG. 1. (a) Layout of IOTA; electrons circulate clockwise. (b) Light path from the undulator to the detector (not to scale). (c) Block diagram of the detection circuit; $i$ is the IOTA revolution number. The delay in the comb filter equals exactly one IOTA revolution, 133.3 ns. Hence, its $\Delta$ channel provides a signal difference between two consecutive turns. 
electron of the electron bunch was $9.1 \times 10^{-3}$. The empirical value was $8.8 \times 10^{-3}$ [22]. There were no free adjustable parameters in this calculation.

Figure 1(c) illustrates our photodetection circuit. First, the radiation pulse is converted into a photocurrent pulse by the photodiode. Then, the photocurrent pulse is integrated by an op-amp-based $R C$ integrator and converted to a voltage signal $A_{i} f(t)$, where $A_{i}$ is the signal amplitude at the $i$ th turn and $f(t)$ is the average signal for one turn, normalized so that its maximum value is 1 . The number of detected photons (photoelectrons) at the $i$ th turn can be obtained as

$$
\mathcal{N}_{i}=\chi A_{i},
$$

where $\chi=2.08 \times 10^{7}$ photoelectrons/V, with a $5 \%$ uncertainty, as per the characteristics of our integrator and the photodiode [22]. The op-amp was capable of driving the 50- $\Omega$ input load of a fast digitizing scope, located $\approx 100 \mathrm{~m}$ away. In our measurements, $A_{i} \in[0,1.2] \mathrm{V}$.

The expected relative fluctuation of $A_{i}$ was $10^{-4}-10^{-3}$ (rms), which is considerably lower than the digitization resolution of our 8-bit broadband oscilloscope. To overcome this problem, we employed a passive comb (notch) filter [27]; see Fig. 1(c). In this filter, the input signal first passes through a two-way splitter. One arm is delayed relative to the other by exactly one IOTA revolution. Then, the difference and the sum of the two signals are produced in the output $\Delta$ and $\Sigma$ channels. For an ideal comb filter,

$$
\begin{aligned}
& \Delta_{i}(t)=\xi\left(A_{i}-A_{i-1}\right) f(t), \\
& \Sigma_{i}(t)=\xi\left(A_{i}+A_{i-1}\right) f(t) .
\end{aligned}
$$

In our filter, $\xi=0.31$, which was measured by comparing input and output pulses. Now, since the offset was removed [Eq. (5)], we were able to directly observe the submillivolt turn-to-turn fluctuations in the $\Delta$ channel and the oscilloscope operated in the appropriate scale setting with negligible digitization noise.

For each measurement, we recorded 1.5-ms-long waveforms (about 11250 IOTA revolutions) of $\Delta$ and $\Sigma$ channels with the oscilloscope at $20 \mathrm{GSa} / \mathrm{s}$. The beam current decay was negligible during the acquisition time. The photoelectron count variance $\operatorname{var}(\mathcal{N})$ and the photoelectron count mean $\langle\mathcal{N}\rangle$ were obtained from the 11250 collected amplitudes, $\Delta_{i}\left(t_{\text {peak }}\right)$ and $\Sigma_{i}\left(t_{\text {peak }}\right)$, as

$$
\begin{gathered}
\operatorname{var}(\mathcal{N})=\chi^{2} \operatorname{var}(A)=\frac{\chi^{2} \operatorname{var}\left(\Delta\left(t_{\text {peak }}\right)\right)}{2 \xi^{2}}, \\
\langle\mathcal{N}\rangle=\chi\langle A\rangle=\chi \frac{\left\langle\Sigma\left(t_{\text {peak }}\right)\right\rangle}{2 \xi},
\end{gathered}
$$

where $t_{\text {peak }}$ is the time within each turn corresponding to the peak of the signal, $f\left(t_{\text {peak }}\right)=1$. These formulas follow from Eqs. (4)-(6). There was a small cross talk $(<1 \%)$ between the output channels of the comb filter. However, its effect is negligible in Eqs. (7) and (8). Also, there was some instrumental noise contribution to $\operatorname{var}\left(\Delta\left(t_{\text {peak }}\right)\right)$. Its contribution to $\operatorname{var}(\mathcal{N})$ was $2.0 \times 10^{8}$, as measured at zero beam current. Primary sources of this noise were the integrator's op-amp and the oscilloscope's pre-amp. In Ref. [22], we showed that this noise level was independent of $\langle\mathcal{N}\rangle$ via measurements with an independent test light source. Therefore, it can be simply subtracted. Reference [22] also describes the details of the photocurrent integrator and the comb filter.

The number of coherent modes $M$ and, hence, the fluctuations $\operatorname{var}(\mathcal{N})$ depend on the following bunch parameters: $\epsilon_{x}, \epsilon_{y}$ (or mode emittances $\left.\epsilon_{1}, \epsilon_{2}\right), \sigma_{p}, \sigma_{z}^{\text {eff }}$. When only one of them is unknown and $\operatorname{var}(\mathcal{N})$ is known (or measured), we can numerically solve Eq. (1), using our general formula for $M$ [[22] Eq. (2)], to find the unknown bunch parameter. Below we consider two such situations.

In the first case, we consider a strongly coupled $[28,29]$ transverse focusing optics in IOTA, which was specifically designed to keep the two mode emittances equal $\epsilon_{1}=\epsilon_{2}=\epsilon$. This was empirically confirmed to be true with a few percent precision. We will call this setup "round beam." The longitudinal bunch profile was measured by a high-bandwidth wall-current monitor [30] to determine $\sigma_{z}^{\text {eff }}$ and estimate $\sigma_{p}$. The fluctuations $\operatorname{var}(\mathcal{N})$, measured using Eq. (7), are shown in Fig. 2(a), with a statistical error of $2.7 \times 10^{6}$ (at all beam currents), which was determined with an independent test light source [22]. Hence, the only unknown parameter in Eq. (1) is $\epsilon$. The numerical solution of Eq. (1) with $M$ from Eq. (2) of Ref. [22] was performed on the Midway2 cluster at the University of Chicago Research Computing Center. The results for $\epsilon$ are shown in Fig. 2(c) (red points). The error bars correspond to the statistical error of the fluctuations measurement. Apart from this statistical error there is also a systematic error due to the $1 \mathrm{MeV}$ uncertainty on the beam energy (from $10 \mathrm{~nm}$ at lower beam currents to $14 \mathrm{~nm}$ at higher currents).

In IOTA, transverse beam sizes are monitored by seven SLMs, at M1L-M4L and at M1R-M3R; see Fig. 1(a). Beam emittances can be determined from the measured sizes using the design Twiss functions. Such measurements for $\epsilon$ of the round beam [blue line in Fig. 2(c)] agree with the fluctuations-based $\epsilon$ within the uncertainties. The smallest reliably resolvable emittance by the SLMs in our experiment configuration was $\approx 20 \mathrm{~nm}$. The measured round-beam emittance $\epsilon$ is $75-100 \mathrm{~nm}$ (rms, unnormalized), primarily due to intrabeam scattering $[31,32]$. The expected zero-current value is $\epsilon \approx 12 \mathrm{~nm}$.

In the second case, we consider uncoupled focusing, with the vertical emittance much smaller than the horizontal one. We will call this configuration "flat beam." 

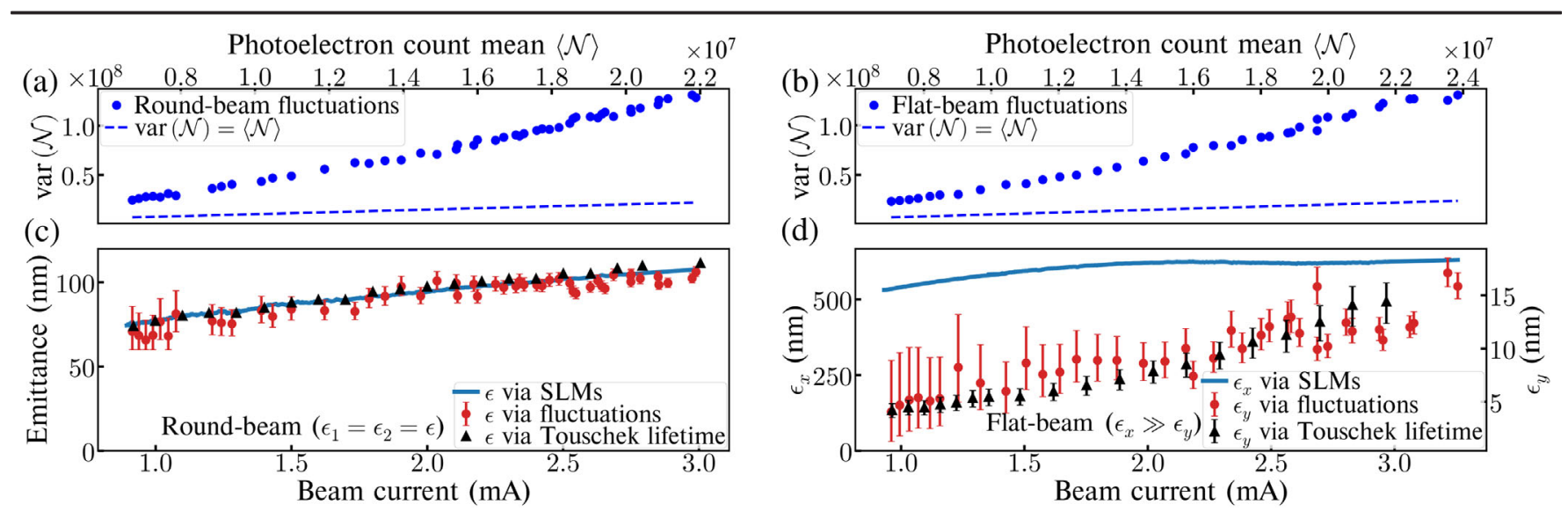

FIG. 2. Panels (a) and (b) show the measured fluctuations for the round and flat beams, respectively. The statistical error of each point is $2.7 \times 10^{6}$ (not shown). (c) The round-beam mode emittance $\epsilon$, determined via SLMs, via undulator radiation fluctuations, and via Touschek lifetime, assuming the effective momentum acceptance $2.0 \times 10^{-3}$. (d) The flat-beam horizontal emittance measurement via SLMs (left-hand scale), the vertical emittance measurement via fluctuations and via Touschek lifetime (right-hand scale). The SLMs had a monitor-to-monitor spread of $\pm 8 \mathrm{~nm}$ (round beam) and $\pm 50 \mathrm{~nm}$ (horizontal emittance of flat beam); these error bars are not shown. All emittances are rms, unnormalized.

The horizontal emittance $\epsilon_{x}$ of the flat beam can still be reliably measured by the SLMs; $\sigma_{z}^{\text {eff }}$ and $\sigma_{p}$ can still be measured by the wall-current monitor. However, the seven SLMs provided very inconsistent estimates for the much smaller $\epsilon_{y}$-the max-to-min variation for different SLMs reached a factor of 8 . We believe this happened because the beam images were close to the resolution limit, set by a combination of factors, such as the diffraction limit, the point spread function of the cameras, chromatic aberrations, the effective radiator size of the dipole magnet radiation $(\approx 20 \mu \mathrm{m})$, and the camera pixel size $(\approx 10 \mu \mathrm{m}$ in terms of beam size). Therefore, the monitor-to-monitor emittance variation primarily came from the Twiss betafunction variation $\left(\beta_{y}^{(\max )} / \beta_{y}^{(\min )} \approx 12\right)$. Although the resolution of the SLMs may be improved in the future [22], at present, $\epsilon_{y}$ of the flat beam is unresolvable by the SLMs, and, therefore, is truly unknown. However, the measured fluctuations for the flat beam, shown in Fig. 2(b), were of the same order as for the round beam, with the same statistical error. Hence, we were able to reconstruct $\epsilon_{y}$ in the same way as $\epsilon$ in Fig. 2(c). The results are shown in Fig. 2(d) (red points, right-hand vertical scale) along with the SLMs data for $\epsilon_{x}$ (blue line, left-hand vertical scale). In addition to the statistical error of $\epsilon_{y}$, shown in Fig. 2(d), there was also a systematic error due to the $1 \mathrm{MeV}$ uncertainty on the beam energy (from $2.5 \mathrm{~nm}$ at lower currents to $5 \mathrm{~nm}$ at higher currents), and a systematic error due to the $50 \mathrm{~nm}$ uncertainty on $\epsilon_{x}$ (from $1.3 \mathrm{~nm}$ at lower currents to $2.4 \mathrm{~nm}$ at higher currents). The measured vertical emittance is $5-15 \mathrm{~nm}$, most likely due to a nonzero residual transverse coupling. The expected zero-current flat-beam emittances were $\epsilon_{x} \approx 50 \mathrm{~nm}, \epsilon_{y} \gtrsim$ $0.33 \mathrm{pm}$ (set by the quantum excitation in a perfectly uncoupled ring).
The vertical emittance $\epsilon_{y}$ of the flat beam in IOTA could also be estimated from the measured beam lifetime $|I /(\mathrm{d} I / \mathrm{d} t)|$, assuming that it is determined solely by Touschek scattering [33], which is a good approximation at beam currents $I \gtrsim 0.5 \mathrm{~mA}$ [29]. In storage rings, the Touschek lifetime is determined by the effective momentum acceptance $\delta_{\text {acc }}^{\text {(eff) }}$ [34], which is smaller than or equal to the rf bucket half-height, $\delta_{\mathrm{rf}}=2.8 \times 10^{-3}$ in IOTA. We measured the IOTA beam lifetime (550-1000 s) for both round and flat beams as a function of beam current. Using the known round-beam emittance and the bunch length, we arrived at the following estimate for IOTA, $\delta_{\text {acc }}^{(\text {eff })}=2.0 \times 10^{-3}$, by comparing the calculated $[35,36]$ Touschek lifetime and the measured beam lifetime (for details, see Appendix D of Ref. [22]). The black triangles in Fig. 2(c) illustrate the emittance of the round beam $\epsilon$, determined from the measured beam lifetime using the Touschek lifetime calculation with $\delta_{\text {acc }}^{(\text {eff })}=2.0 \times 10^{-3}$. Then, we used this value of $\delta_{\text {acc }}^{(\text {eff })}$ and the values of $\epsilon_{x}$, measured by the SLMs, to estimate the vertical emittance $\epsilon_{y}$ of the flat beam via the Touschek lifetime. The results are shown in Fig. 2(d) (black triangles). The error bars correspond to the $\pm 50 \mathrm{~nm}$ uncertainty on $\epsilon_{x}$.

During our measurements, the rms and the effective bunch lengths $\sigma_{z}, \sigma_{z}^{\text {eff }}$ were $26-31 \mathrm{~cm}$ and $24-30 \mathrm{~cm}$, respectively, primarily due to intrabeam scattering. They were different because the longitudinal bunch shape was not exactly Gaussian due to beam interaction with its environment [37]. The relative rms momentum spread was $\sigma_{p} \approx 9.1 \times 10^{-6} \times \sigma_{z}[\mathrm{~cm}]$, based on the $\mathrm{rf}$ cavity and ring parameters. The expected zero-current values are $\sigma_{z}=\sigma_{z}^{\text {eff }}=9 \mathrm{~cm}, \sigma_{p}=8.3 \times 10^{-5}$. The uncoupled case Twiss beta functions in the undulator were $\beta_{x}=204 \mathrm{~cm}$, $\beta_{y}=98 \mathrm{~cm}$; for more details, see Ref. [22]. 
Other emittance monitors (wire scanners, Comptonscattering monitors $[38,39]$ ) could provide better resolution in IOTA. However, if a bright synchrotron light source is available, our fluctuations-based monitor may be a good inexpensive noninvasive alternative. There are two requirements for the technique to work: (A) the fluctuations should not be dominated by the Poisson noise, so that $M$ can be reliably deduced from $\operatorname{var}(\mathcal{N})$, and (B) $M$ has to be sensitive to $\epsilon_{x}, \epsilon_{y}$. Let us consider the $h$ th harmonic of undulator radiation in the approximation of Eqs. (2) and (3) with a narrow Gaussian filter $\sigma_{k} \ll k_{0} /\left(h N_{\mathrm{u}}\right)$ and $k_{0}=2 \pi h / \lambda_{1}$. By integrating Eq. (2) we obtain $\langle\mathcal{N}\rangle=C(2 \pi)^{3 / 2} \sigma_{\theta_{x}} \sigma_{\theta_{y}} \sigma_{k}$, where $C$ is the peak on-axis photon flux, $C=\alpha N_{\mathrm{u}}^{2} \gamma^{2} F_{h}\left(K_{\mathrm{u}}\right) n_{e} / k_{0}$ [[26] p. 68], $\alpha$ is the fine-structure constant, $n_{e}$ is the number of electrons per bunch, and the function $F_{h}\left(K_{\mathrm{u}}\right)$, defined in Ref. [26] p. 69, is typically about $0.2-0.4$. If we approximate Eq. (3) by $M \approx 8 k_{0}^{2} \sigma_{k} \sigma_{\theta_{x}} \sigma_{\theta_{v}} \sigma_{x} \sigma_{y} \sigma_{z}$, the requirement (A) becomes [see Eq. (1)]

$$
\frac{\langle\mathcal{N}\rangle}{M}=\alpha\left(\frac{\pi}{2}\right)^{3 / 2} F_{h}\left(K_{u}\right) \frac{\gamma^{2} N_{\mathrm{u}}^{2} n_{e}}{\sigma_{x} \sigma_{y} \sigma_{z} k_{0}^{3}} \gtrsim 1 .
$$

In the model of Eq. (3), the requirement (B) becomes $\sigma_{x} \gtrsim 1 /\left(2 k_{0} \sigma_{\theta_{x}}\right), \sigma_{y} \gtrsim 1 /\left(2 k_{0} \sigma_{\theta_{y}}\right)$. Notably, one can intentionally make $M$ insensitive to $\sigma_{x}$ (or $\sigma_{y}$ ), and, thus, enable an independent measurement of $\sigma_{y}\left(\right.$ or $\left.\sigma_{x}\right)$. For example, by using a vertical slit, which can be approximated by a very small $\sigma_{\theta_{x}} \ll 1 /\left(2 k_{0} \sigma_{x}\right)$, one can deduce $\sigma_{y}$ from a measured $M$ without the knowledge of $\sigma_{x}$. Also, radiation masks can be applied to analyze fluctuations in various portions of the angular distribution of the radiation, which adds flexibility to this method. Assuming no angular restrictions, $\sigma_{\theta_{x}}, \sigma_{\theta_{y}} \approx \sqrt{\lambda_{0} /\left(2 L_{\mathrm{u}}\right)}$ [[17] Eq. (2.57)], and the requirement $(\mathrm{B})$ becomes

$$
\sigma_{x}, \sigma_{y} \gtrsim \sqrt{2 L_{\mathrm{u}} \lambda_{0}} /(4 \pi),
$$

where $\lambda_{0}=2 \pi / k_{0}, L_{\mathrm{u}}$ is the undulator length. In IOTA, this corresponds to $\sigma_{x}, \sigma_{y} \gtrsim 50 \mu \mathrm{m}$, or $\epsilon_{x} \gtrsim 1.0 \mathrm{~nm}, \epsilon_{y} \gtrsim$ $2.2 \mathrm{~nm}$, and $\langle\mathcal{N}\rangle / M \in[2.3,5.0]$ (as per measurements).

Equation (10) shows that the resolution limit improves with a shorter wavelength. Therefore, this technique may be particularly beneficial for existing state-of-the-art and next generation low-emittance high-brightness ultraviolet and x-ray synchrotron light sources. Consider the Advanced Photon Source Upgrade (APS-U) with a round beam configuration, for example. The beam energy is $6 \mathrm{GeV}, n_{e}=9.6 \times 10^{10}$, $\sigma_{z}=3.1 \mathrm{~cm}, \epsilon_{x}=31.9 \mathrm{pm}, \epsilon_{y}=31.7 \mathrm{pm}, \sigma_{x}=12.9 \mu \mathrm{m}$, $\sigma_{y}=8.7 \mu \mathrm{m}, \quad \sigma_{x^{\prime}}=2.5 \mu \mathrm{rad}, \quad \sigma_{y^{\prime}}=3.6 \mu \mathrm{rad} \quad[40]$. Let us use the fundamental harmonic $\lambda_{1}=4.1 \AA$ of the undulator with $\lambda_{\mathrm{u}}=28 \mathrm{~mm}, K_{\mathrm{u}}=2.459$, and $L_{\mathrm{u}}=2.1 \mathrm{~m}$. Equation (9) yields $\langle\mathcal{N}\rangle / M=19$, and
Eq. (10) becomes $\sigma_{x}, \sigma_{y} \gtrsim 3.3 \mu \mathrm{m}$. Thus, both requirements (A) and (B) are satisfied. These estimates were confirmed by Eqs. (2)-(8) of Ref. [22].

We would like to thank the entire FAST/IOTA team at Fermilab for helping us with building and installing the experimental setup and taking data, especially Mark Obrycki, James Santucci, and Wayne Johnson. Greg Saewert constructed the detection circuit and provided the test light source. Brian Fellenz, Daniil Frolov, David Johnson, and Todd Johnson provided equipment and assisted during our detector tests. This work was completed in part with resources provided by the University of Chicago Research Computing Center. This research is supported by the University of Chicago and the U.S. Department of Energy under Contracts No. DE-AC0276SF00515 and No. DE-AC02-06CH11357. This manuscript has been authored by Fermi Research Alliance, LLC under Contract No. DE-AC02-07CH11359 with the U.S. Department of Energy, Office of Science, Office of High Energy Physics.

*ilobach@uchicago.edu

${ }^{\dagger}$ Also at The Enrico Fermi Institute, The University of Chicago, Chicago, Illinois 60637, USA.

[1] J. B. Johnson, Thermal agitation of electricity in conductors, Phys. Rev. 32, 97 (1928).

[2] A. W. Hull and N. Williams, Determination of elementary charge $e$ from measurements of shot-effect, Phys. Rev. 25, 147 (1925).

[3] S. van der Meer, Stochastic cooling and the accumulation of antiprotons, Nobel Lecture (1984), https://www.nobelprize .org/uploads/2018/06/meer-lecture.pdf.

[4] W. Schottky, Über spontane Stromschwankungen in verschiedenen Elektrizitätsleitern, Ann. Phys. (Berlin) 362, 541 (1918).

[5] D. Boussard, Schottky noise and beam transfer function diagnostics, CERN Technical Report No. CERN-SPS-8611-ARF, 1986.

[6] S. van der Meer, Diagnostics with Schottky noise, in Frontiers of Particle Beams; Observation, Diagnosis and Correction (Springer, New York, 1989), pp. 423-433.

[7] F. Caspers, J. M. Jimenez, O. R. Jones, T. Kroyer, C. Vuitton, T. W. Hamerla, A. Jansson, J. Misek, R. J. Pasquinelli, P. Seifrid et al., The $4.8 \mathrm{GHz}$ LHC Schottky pick-up system, in Proceedings of the 2007 IEEE Particle Accelerator Conference (PAC) (IEEE, New York, 2007), pp. 4174-4176.

[8] M. C. Teich, T. Tanabe, T. C. Marshall, and J. Galayda, Statistical Properties of Wiggler and Bending-Magnet Radiation from the Brookhaven Vacuum-Ultraviolet Electron Storage Ring, Phys. Rev. Lett. 65, 3393 (1990).

[9] M. S. Zolotorev and G. V. Stupakov, Fluctuational interferometry for measurement of short pulses of incoherent radiation, SLAC Technical Report No. SLAC-PUB-7132, 1996. 
[10] F. Sannibale, G. V. Stupakov, M. S. Zolotorev, D. Filippetto, and L. Jägerhofer, Absolute bunch length measurements by incoherent radiation fluctuation analysis, Phys. Rev. ST Accel. Beams 12, 032801 (2009).

[11] V. Sajaev, Measurement of bunch length using spectral analysis of incoherent radiation fluctuations, AIP Conf. Proc. 732, 73 (2004).

[12] V. Sajaev, Determination of longitudinal bunch profile using spectral fluctuations of incoherent radiation, Argonne National Laboratory Report No. ANL/ASD/CP-100935, 2000.

[13] P. Catravas, W. P. Leemans, J. S. Wurtele, M. S. Zolotorev, M. Babzien, I. Ben-Zvi, Z. Segalov, X.-J. Wang, and V. Yakimenko, Measurement of Electron-Beam Bunch Length and Emittance Using Shot-Noise-Driven Fluctuations in Incoherent Radiation, Phys. Rev. Lett. 82, 5261 (1999).

[14] S. Antipov, D. Broemmelsiek, D. Bruhwiler, D. Edstrom, E. Harms, V. Lebedev, J. Leibfritz, S. Nagaitsev, C.-S. Park, H. Piekarz et al., IOTA (Integrable Optics Test Accelerator): Facility and experimental beam physics program, J. Instrum. 12, T03002 (2017).

[15] N. Kuklev, J. Jarvis, Y. Kim, A. Romanov, J. Santucci, and G. Stancari, Synchrotron radiation beam diagnostics at IOTACommissioning performance and upgrade efforts, in Proceedings of the 10th International Particle Accelerator Conference (IPAC'19), Melbourne, Australia, 2019 (JACoW Publishing, Geneva, Switzerland, 2019), pp. 2732-2735, https://doi.org/10.18429/JACoW-IPAC2019-WEPGW103.

[16] I. Lobach, V. Lebedev, S. Nagaitsev, A. Romanov, G. Stancari, A. Valishev, A. Halavanau, Z. Huang, and K.-J. Kim, Statistical properties of spontaneous synchrotron radiation with arbitrary degree of coherence, Phys. Rev. Accel. Beams 23, 090703 (2020).

[17] K.-J. Kim, Z. Huang, and R. Lindberg, Synchrotron Radiation and Free-Electron Lasers (Cambridge University Press, Cambridge, England, 2017).

[18] J.-W. Park, An investigation of possible non-standard photon statistics in a free-electron laser, Ph. D. thesis, University of Hawaii at Manoa, 2019.

[19] R. J. Glauber, The quantum theory of optical coherence, Phys. Rev. 130, 2529 (1963).

[20] R. J. Glauber, Coherent and incoherent states of the radiation field, Phys. Rev. 131, 2766 (1963).

[21] R. J. Glauber, Some notes on multiple-boson processes, Phys. Rev. 84, 395 (1951).

[22] I. Lobach, S. Nagaitsev, V. Lebedev, A. Romanov, G. Stancari, A. Valishev, A. Halavanau, Z. Huang, and K.-J. Kim, companion paper, Measurements of undulator radiation power noise and comparison with $a b$ initio calculations, Phys. Rev. Accel. Beams 24, 040701 (2021).

[23] I. Lobach, The source code for calculation of fluctuations in wiggler radiation, https://github.com/IharLobach/fur.
[24] Hamamatsu InGaAs PIN photodiode G11193-10R, https:// www.hamamatsu.com/us/en/product/type/G11193-10R/ index.html, accessed 2020-11-18.

[25] I. Lobach, The source code for calculation of spectralangular distribution of wiggler radiation, https://github.com/ IharLobach/wigrad.

[26] J. A. Clarke, The Science and Technology of Undulators and Wigglers (Oxford University Press on Demand, New York, 2004), pp. 66-67.

[27] J. O. Smith, Physical Audio Signal Processing: For Virtual Musical Instruments and Audio Effects (W3K Publishing, 2010), http://www.w3k.org/.

[28] V. A. Lebedev and S. Bogacz, Betatron motion with coupling of horizontal and vertical degrees of freedom, J. Instrum. 5, P10010 (2010).

[29] V. Lebedev, Report on single and multiple intrabeam scattering measurements in IOTA ring in Fermilab, Fermilab Report No. FERMILAB-TM-2750-AD, 2020.

[30] B. Fellenz and J. Crisp, An improved resistive wall monitor, AIP Conf. Proc. 451, 446 (1998).

[31] J. D. Bjorken and S. K. Mtingwa, Intrabeam scattering, Part. Accel. 13, 115 (1982).

[32] S. Nagaitsev, Intrabeam scattering formulas for fast numerical evaluation, Phys. Rev. ST Accel. Beams 8, 064403 (2005).

[33] C. Bernardini, G. F. Corazza, G. Di Giugno, G. Ghigo, J. Haissinski, P. Marin, R. Querzoli, and B. Touschek, Lifetime and Beam Size in a Storage Ring, Phys. Rev. Lett. 10, 407 (1963).

[34] N. Carmignani, Touschek lifetime studies and optimization of the european synchrotron radiation facility, $\mathrm{Ph} . \mathrm{D}$. thesis, Pisa University, 2014, pp. 25-26.

[35] V. Lebedev, Intrabeam scattering, in Handbook of Accelerator Physics and Engineering, edited by A. Chao, K. Mess, M. Tigner, and F. Zimmermann (World Scientific, Singapore, 2013), pp. 155-158.

[36] A. Piwinski, The Touschek effect in strong focusing storage rings, arXiv:physics/9903034.

[37] J. Haïssinski, Exact longitudinal equilibrium distribution of stored electrons in the presence of self-fields, Nuovo Cimento Soc. Ital Fis. B 18, 72 (1973).

[38] P. Tenenbaum and T. Shintake, Measurement of small electron-beam spots, Annu. Rev. Nucl. Part. Sci. 49, 125 (1999).

[39] H. Sakai, Y. Honda, N. Sasao, S. Araki, Y. Higashi, T. Okugi, T. Taniguchi, J. Urakawa, and M. Takano, Measurement of an electron beam size with a laser wire beam profile monitor, Phys. Rev. ST Accel. Beams 4, 022801 (2001).

[40] Advanced photon source upgrade project, Final design report, Argonne National Laboratory Technical Report No. APSU-2.01-RPT-003, 2019, Chap. 2, Table 2.1; Chap. 4, Table 4.62. 